\title{
Binary toxin locus analysis in Clostridium difficile
}
Correspondence
Devon S. Metcalf
dmetcalf@uoguelph.ca

Received 28 November 2010

Accepted 28 March 2011

\author{
Devon S. Metcalf and J. Scott Weese
}

\author{
Department of Pathobiology, Ontario Veterinary College, University of Guelph, Guelph, \\ ON N1G 2W1, Canada
}

\begin{abstract}
The objective of this study was to compare full binary toxin loci (CDTloc) sequences from a collection of Clostridium difficile isolates in an effort to further understand the regulation of the binary toxin $(\mathrm{Cdt} A B)$ and its putative regulator $(\mathrm{CdtR})$. Sequences from different ribotypes and toxinotypes were analysed phylogenetically and for polymorphisms, non-sense mutations, promoter features and signal sequences. Expression of $c d t A$, which was also representative of $c d t B$ expression, was measured by quantitative PCR (qPCR). Several consensus promoter features and various polymorphisms were identified including a non-sense mutation identified in a ribotype $078 \mathrm{cdtR}$ gene that is predicted to result in a severely truncated protein. Despite this mutation, $c d t A$ expression was still detected by qPCR. Dendrograms based on total sequences indicated that isolates belonging to the same ribotype shared the greatest similarity within the binary toxin locus. Although $c d t R$ is thought to be involved in regulation of $\operatorname{cdt} A$ expression, a $c d t R$ non-sense mutation did not inhibit expression of $c d t A$, suggesting that either the truncated protein is functional or another regulator of the binary toxin exists.
\end{abstract}

\section{INTRODUCTION}

Clostridium difficile is a Gram-positive spore-forming anaerobe and a common cause of antimicrobial- and hospital-associated diarrhoea in humans. The increase in frequency and severity of $C$. difficile infection (CDI), largely due to the emergence of hypervirulent ribotypes, has rendered this pathogen a significant and growing healthcare concern (McDonald et al., 2005). C. difficile has two main virulence factors, toxin $A(\operatorname{Tcd} A)$ and toxin $B(\operatorname{TcdB})$, which cause deregulation of intestinal cell function by glucosylating the Rho subfamily of proteins (Just et al., 1994, 1995). A third infrequently studied toxin, the binary toxin (CDT), is produced by many toxigenic strains. It consists of an enzymic and a binding component, and has been shown to have cytotoxic properties (Perelle et al., 1997). This toxin is homologous to the iota toxin of Clostridium perfringens and functions as an ADP-ribosyltransferase (Gülke et al., 2001). Although its role in pathogenesis is poorly understood, it has been shown to promote adhesion by the induction of microbtubule-based protrusions in intestinal epithelial cells (Schwan et al., 2009).

Full-length CDT sequences are commonly found in variant toxinotypes (those with significant polymorphisms in the pathogenicity locus encoding $t c d A$ and $t c d B$ ), whereas nonvariant toxinotypes often have a common truncation in the CDT locus resulting in a binary toxin-negative phenotype

Abbreviations: APSSP, advanced protein secondary structure prediction; $\mathrm{CDI}$, Clostridium difficile infection; qPCR, quantitative real-time PCR; SNP, single nucleotide polymorphism.

The GenBank/EMBL/DDBJ accession numbers for the sequences determined in this study are H0639670-H0639679.
(Stubbs et al., 2000; Stare et al., 2007). Analysis of the CDT locus has revealed $c d t A$ to be more conserved than $c d t B$ suggesting a possible enhanced mutability of the gene encoding the binding component (Pituch et al., 2005).

Few studies investigating gene expression in $C$. difficile have been performed (Gerber et al., 2008; Govind et al., 2009; Karlsson et al., 2008) and, as a result, little is known about the regulation of genes, virulence-related or otherwise. With respect to the binary toxin, Carter et al. (2007) demonstrated expression of $c d t A$ during the stationary growth phase and expression was significantly reduced in strains with a mutation in $c d t R$, a gene encoding a putative regulator. Promoter analysis is also rarely performed and is usually restricted to genes within the pathogenicity locus. An active promoter, a Shine-Dalgarno consensus sequence and two putative -10 and -35 sequences have been identified upstream of $t c d B$ (Song \& Faust, 1998). Mani et al. (2002) also described putative promoters for $t c d R, t c d A$ and $t c d B$.

To date, no significant analysis of the binary toxin locus (including promoters for $c d t R$ and $c d t A$, and the $c d t R, c d t A$ and $c d t B$ genes) has been performed. The objectives of this study were to sequence, analyse and compare full binary toxin loci (CDTloc) sequences in ten $C$. difficile binary toxin-positive ribotypes, pulsotypes or toxinotypes, then to analyse the binary toxin loci promoters and genes and to perform a phylogenetic analysis of the loci.

\section{METHODS}

C. difficile isolate selection and culture conditions. Isolates were obtained from a collection of $C$. difficile isolates from Ontario, 
Canada, from humans with CDI, collected from 2004 to 2006 (Martin et al., 2008), and an isolate from a diarrhoeic pig collected in 2002. Ten isolates were chosen, representing six different binary toxinpositive ribotypes, four different toxinotypes and six different pulsotypes (Table 1). Isolates belonging to the same ribotype were epidemiologically unrelated. All isolates possessed $t c d A$ and $t c d B$. All C. difficile strains were cultured on Colombia blood agar plates (Oxoid) and incubated anaerobically for $24 \mathrm{~h}$ at $37^{\circ} \mathrm{C}$.

Nucleic acid extraction and PCR amplification. DNA was extracted using a commercial kit following the manufacturer's instructions (Instagene Matrix; Bio-Rad). The 28 primers listed in Table 2 were used to sequence the binary toxin locus including the $c d t R$ promoter, $c d t R$, the $c d t A B$ promoter, $c d t A$ and $c d t B$. Briefly, fragments of the locus were amplified in a Techne Touchgene Gradient thermocycler (Dynalab) using the following PCR cycling parameters: $94{ }^{\circ} \mathrm{C}$ for $5 \mathrm{~min} ; 30$ cycles of $94{ }^{\circ} \mathrm{C}$ for $60 \mathrm{~s}, 55-62{ }^{\circ} \mathrm{C}$ for $60 \mathrm{~s}, 72{ }^{\circ} \mathrm{C}$ for $90 \mathrm{~s}$; and a final extension of $5 \mathrm{~min}$ at $72{ }^{\circ} \mathrm{C}$. The reaction mixtures consisted of $1 \times$ PCR buffer, $1-4 \mathrm{mM} \mathrm{MgCl}_{2}$, $250 \mu \mathrm{M}$ dNTPs, $10 \mathrm{pmol}$ of each primer, $1 \mathrm{U}$ Taq polymerase and $100 \mathrm{mM}$ tetramethylammonium. PCR products were purified using a QIAquick PCR purification kit (Qiagen) and sequenced (Macrogen). Ribotyping was performed as described by Bidet et al. (1999). PCR products were resolved on a $1.5 \%$ agarose gel and visualized by ethidium bromide staining.

CDTloc analysis. Binary toxin locus sequence processing and assembly was performed using the Geneious bioinformatics software (Biomatters, Auckland, New Zealand). Signal sequences and their respective cleavage sites were predicted using the SignalP 3.0 server (http://www.cbs.dtu.dk/services/SignalP/). Molecular masses and isoelectric points were predicted using ProtParam (Gasteiger et al., 2005; http://www.expasy.ch/tools/protparam.html). Multiple sequence alignments were performed using the CLUSTAL_w2 tool (http://www. ebi.ac.uk/Tools/msa/clustalw2/) and phylogenetic trees were created using CLUSTAL_X version 2.0 using the neighbour-joining method. Secondary structure prediction was performed using the advanced protein secondary structure prediction (APSSP) server (http://imtech. res.in/raghava/apssp/).

Quantitative real-time PCR (qPCR) analysis of cdtR and cdtA expression. qPCR analysis of the binary toxin and regulator genes was performed on the ten $C$. difficile strains listed in Table 1 . These strains were cultured anaerobically at $37{ }^{\circ} \mathrm{C}$ in a $\mathrm{C}$. difficile growth medium broth consisting of $\left(\mathrm{g} \mathrm{l}^{-1}\right.$, unless otherwise stated): proteose peptone, 40; disodium hydrogen phosphate, 5.0; potassium dihydrogen phosphate, 1.0; magnesium sulfate, 0.1 ; sodium chloride, 2.0; fructose, 6.0; and sodium taurocholate, $0.1 \%(\mathrm{w} / \mathrm{v})$. Ten millilitres of exponential phase $\left(\mathrm{OD}_{600} \quad 0.3\right)$ and stationary phase $\left(\mathrm{OD}_{600}\right.$ 1.0) cultures were pelleted, resuspended in $500 \mu \mathrm{l}$ RNAlater RNA stabilization reagent (Ambion) and frozen at $-80{ }^{\circ} \mathrm{C}$ until use. RNA was extracted using Maxwell 16, a robotic, magnetic bead-based system (Promega). Isolates were grown and RNA samples were extracted in triplicate. Samples were treated with the Turbo DNA-free system (Ambion) and subjected to a reverse transcription reaction using the Omniscript Reverse Transcription kit (Qiagen) and gene-specific primers. qPCR was performed using SYBR green to assess expression of $c d t A$ and $c d t R$. Expression was normalized using the reference gene $r p o A$. The $c d t A$ primers used have been described previously (Carter et al., 2007). $c d t R$ and $r p o A$ fragments were amplified using the primer pair described in Table 3.

CdtR analysis by pyrosequencing. A pyrosequencing protocol was developed to screen for a truncating single nucleotide polymorphism (SNP) in the $c d t R$ gene in 57 toxinotype $\mathrm{V}$ isolates (51 ribotype 078 isolates and six from three other ribotypes). In addition, 22 ribotype 027/toxinotype III isolates were screened and seven additional isolates were found to belong to six other ribotypes associated with CDI in humans. Amplification and sequencing primers were designed using Assay Design Software (Qiagen). A 97 bp $c d t R$ fragment was amplified using the forward and the biotinylated reverse pyrosequencing primers (Table 3 ). One sequencing primer was used to sequence over the SNP region. Locations of the PCR and sequencing primers are shown in Fig. 1. The $c d t R$ fragments were amplified using DNA from 78 C. difficile strains using real-time PCR in a LightCycler 1.5 thermocycler (Roche Diagnostics). Reactions $(25 \mu \mathrm{l})$ were prepared using the LightCycler FastStart DNA Master SYBR Green I (Roche Diagnostics) following the manufacturer's instructions. The following thermocycling parameters were used: denaturation at $95{ }^{\circ} \mathrm{C}$ for $5 \mathrm{~min}$; followed by 45 cycles of $10 \mathrm{~s}$ at $95{ }^{\circ} \mathrm{C}, 15 \mathrm{~s}$ at $61{ }^{\circ} \mathrm{C}$ and $15 \mathrm{~s}$ at $72{ }^{\circ} \mathrm{C}$; melting curve analysis at $95{ }^{\circ} \mathrm{C}$ for $60 \mathrm{~s}, 40{ }^{\circ} \mathrm{C}$ for $60 \mathrm{~s}, 55{ }^{\circ} \mathrm{C}$ for $1 \mathrm{~s}$ increasing to $95{ }^{\circ} \mathrm{C}$ for $0 \mathrm{~s}$; and cooling at $40{ }^{\circ} \mathrm{C}$ for $30 \mathrm{~s}$. The PyroMark vacuum Prep workstation, PyroMark ID instrument and Pyro Gold reagents (Qiagen) were used to perform pyrosequencing. Univariable analysis

Table 1. C. difficile isolates used in this study

NAP, North American pulsotype. All isolates were positive for toxins A and B.

\begin{tabular}{|c|c|c|c|c|c|}
\hline Isolate & Source & Ribotype & Pulsotype & Toxinotype & $\begin{array}{c}\text { GenBank accession no. for } \\
\text { sequenced loci }\end{array}$ \\
\hline 16 & Human & 027 & NAP1 & III & HQ639671 \\
\hline 978 & Human & 027 & NAP1 & III & HQ639678 \\
\hline 667 & Human & $\mathrm{AD}$ & NAP1 & III & HQ639670 \\
\hline 70 & Human & 027 & NAP1 & III & HQ639675 \\
\hline 959 & Human & $\mathrm{C}^{*}$ & OVCA $^{*}$ & IX & HQ639673 \\
\hline 765 & Human & $\mathrm{A}^{*}$ & NAP1 & IX & HQ639677 \\
\hline 421 & Human & $\mathrm{C}^{*}$ & $\mathrm{OVCB}^{*}$ & IX & HQ639672 \\
\hline CD98 & Pig & 078 & NAP7 & V & HQ639674 \\
\hline 744 & Human & 078 & NAP8 & $\mathrm{V}$ & HQ639679 \\
\hline 478 & Human & $\mathrm{AM}^{*}$ & OVCC $^{*}$ & IV & HQ639676 \\
\hline
\end{tabular}

${ }^{\star}$ All ribotypes not internally recognized and all non-NAP types were given an internal laboratory designation. 
Table 2. Primers used to sequence the binary toxin locus

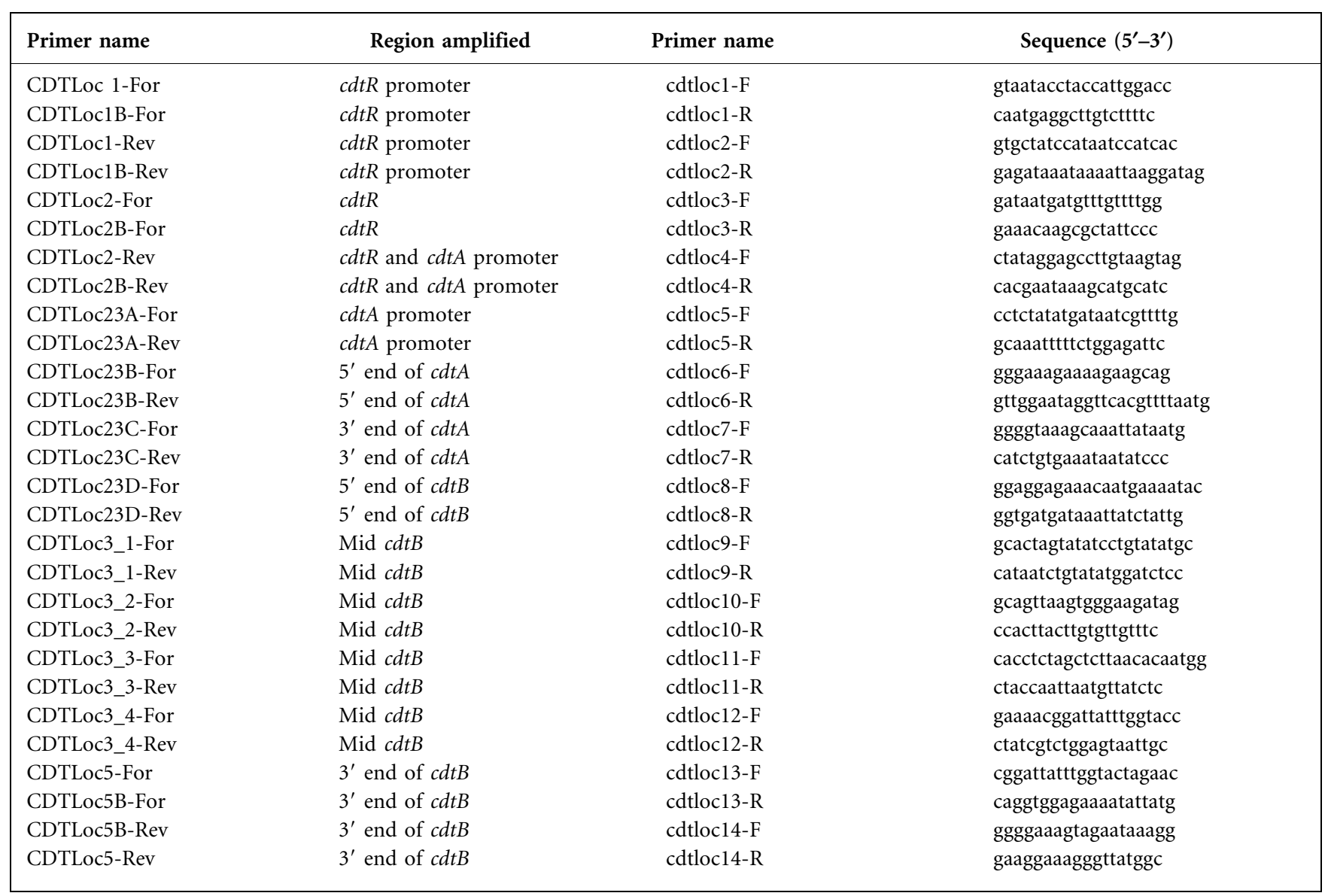

was performed using Fisher's exact test and logistic regression. Nominal logistic regression was used to evaluate the association of year and origin on the presence of the $c d t R$ mutation. $P$ values $<0.05$ were considered significant.

Table 3. Primers used to analyse gene expression and to pyrosequence the $c d t R$ region that contains the truncating SNP

\begin{tabular}{|c|c|}
\hline Primer name & Sequence $\left(5^{\prime}-3^{\prime}\right)$ \\
\hline \multicolumn{2}{|l|}{ qPCR } \\
\hline$c d t A$-For ${ }^{*}$ & tgcaatactacttacaaggctcctataga \\
\hline$c d t A-\operatorname{Rev}^{*}$ & tctttcccattctttagccttttc \\
\hline$c d t R$-For $\dagger$ & cgcaaaatataagataagtaatg \\
\hline$c d t R-\operatorname{Rev} \dagger$ & gcgataatttttttctttaatg \\
\hline rpoA-For ${ }^{*}$ & ggatgatatgatgaaggttagaaacct \\
\hline$r p o A-\operatorname{Rev}^{*}$ & cccaatccaagttcttctagtttttg \\
\hline \multicolumn{2}{|l|}{ Pyrosequencing } \\
\hline$c d t R$-forward & agaaatgattttagggatcttcgat \\
\hline$c d t R$-reverse & biotin-acaagcttttatcttactttcagca \\
\hline$c d t R$-sequence & ttttagggatcttcgatt \\
\hline
\end{tabular}

${ }^{*}$ Carter et al. (2007).

$\dagger$ Metcalf et al. (2010).

\section{RESULTS}

\section{Amplification and sequencing of the CDTloc}

The binary toxin locus, including the putative regulator $(c d t R), \quad c d t A$ and $c d t B$, and their respective promoter regions, was successfully sequenced in ten $C$. difficile isolates. Fourteen primer pairs were needed to overlap and amplify the entire 6082 bp locus.

\section{Prediction of molecular mass, isoelectric point and signal sequences in the ten representative strains}

The predicted CdtR in all isolates consisted of 248 amino acids and a molecular mass of $29.3-29.4 \mathrm{kDa}$, with the exception of 744 (a ribotype 078 isolate). In this isolate, a stop codon was identified after amino acid 107. All predicted CdtA and CdtB protein sequences consisted of 463 and 876 amino acids, respectively. The predicted molecular mass of CdtA for each isolate fell between 53.2 and $53.3 \mathrm{kDa}$ and for $\mathrm{CdtB}$, between 98.7 and $98.8 \mathrm{kDa}$.

The CdtR isoelectric points were calculated to be 9.14 for most sequences. Isolate 98 (ribotype 078) had an isoelectric point of 9.15 and isolate 478 had a point of 9.1. No calculation was made for isolate 744 due to the truncating 


\title{
259 GTCCAATCATATTTCTTACAAATCATATAGAAATGA TTTTAGGGATCTTCGATTATAGGTTAGAATTATGGAC \\ $c d t R$-forward \\ TTTATACTAAAACATGATATGGAAATTGCTGAAAGTAAGATAAAAGCTTGTATTAAAATTGCTCATAAAAGATACA \\ $c d t R$-reverse \\ TTAAAGAAAAAAATTATTGCTCTAAT 435
}

\begin{abstract}
Fig. 1. Design for amplification and pyrosequencing of the $c d t R$ region containing the truncating SNP. The forward and reverse primer regions are indicated by the arrows in the direction of orientation and the sequencing primer is boxed in grey. The location of the SNP is circled.
\end{abstract}

mutation. Greater variation was observed in CdtA. Most isolates had CdtA sequences with isoelectric points measuring 8.84 but the two ribotype 078 isolates had isoelectric points of 8.53 and that of isolate 478 was 8.68. The most variable sequence with respect to isoelectric points was CdtB; isoelectric points of these sequences ranged from 4.92 to 5.05 .

No signal sequences were identified in any CdtR sequences. However, a signal sequence was identified in all CdtA sequences with the predicted cleavage site falling between amino acids 34 and 35. All CdtB sequences also had signal sequences with the cleavage site falling between amino acids 30 and 31 with the exception of isolate 744 (ribotype $\mathrm{K})$. In this isolate, the CdtB signal sequence had a predicted cleavage site between amino acids 26 and 27 .

\section{Identification of promoter features and whole sequence analysis}

The promoter sequences for all isolates listed in Table 1 were also analysed. Approximately $800 \mathrm{nt}$ upstream of $c d t R$ was sequenced and considered to include its promoter. The region between the stop codon of $c d t R$ and the start codon of $c d t A$ was sequenced and considered to include the $c d t A$ promoter. The $c d t R$ promoters of isolates 478 and 744 differed most drastically from the other sequences. Both isolates displayed a $9 \mathrm{nt}$ insertion at position -413. The remaining sequence variations observed consisted of SNPs including substitutions or deletions. The $c d t A$ promoter sequence consisted of $389 \mathrm{nt}$ for all isolates except K98, which had a 1 nt deletion. Fiftytwo nucleotides separated the stop codon of $c d t A$ and the start codon of $c d t B$. During analysis of the sequences, several promoter features were identified. All isolates had a conserved Shine-Dalgarno sequence, AGGAGG, located between nucleotides -13 and -8 of the $c d t R$ promoter (Fig. 2a). A predicted Shine-Dalgarno sequence, GGGAGG, was identified at position -14 to -9 of all $c d t A$ genes sequenced (Fig. 2b). Upstream of the $c d t B$ start codon, a putative ribosome-binding site, AGGAGG, was identified in eight of the ten isolates between nucleotides -13 and -8 (data not shown). Isolate 478 had an AGGGGG sequence at this position and isolate K744 had a predicted Shine-Dalgarno motif, AGGAGG, from nucleotides -25 to -20 . At positions -13 to -8 , the sequence varied from the predicted consensus sequence (AATGAA). The -35 and -10 element consensus sequences (TTGACA and TATAAT, respectively) could not be identified specifically at these positions, but similar sequences were identified further upstream from the start codon (Fig. 2a, b). Alignments of the C. perfringens Ia promoter elements with other $C$. difficile promoters are shown in Fig. 2(c). The -35 and $-10 \sigma^{70}$ consensus sequences are also shown. Most -35 elements shared four of six nucleotides with the $\sigma^{70}$ consensus sequence and, although greater diversity was observed with the -10 element, the $c d t A$ and $c d t R-10$ regions both shared five of six nucleotides with the $-10 \sigma^{70}$ consensus sequence.

Alignments of both gene nucleotide and amino acid sequences were performed. There were numerous SNPs in the $c d t R$ sequence; however, once translated there were only two loci that encoded non-conserved amino acids. There was an R143C polymorphism in isolate 478 and an S153F polymorphism in isolate 98 . It is likely that these polymorphisms would not affect the secondary structure of the protein, as predicted by the APSSP. The isolate 744 $c d t R$ sequence had a truncating stop codon that would result in only 107 versus 248 amino acids being translated. At position 322, an in-frame G322T mutation would introduce the TAA stop codon in place of GAA encoding glutamate.

There was a total of 39 polymorphisms in the $c d t A$ sequence of the ten representative strains, including one double nucleotide polymorphism and one triple nucleotide polymorphism. The majority of these polymorphisms translated into identical or conserved amino acids. The $c d t B$ sequences had a total of 65 polymorphisms including two double nucleotide polymorphisms. Only four polymorphisms resulted in non-identical or non-conserved amino acids. Again, these polymorphisms would probably not affect secondary structure, as predicted by APSSP.

\section{cdtA and cdtR qPCR}

Expression of $c d t A$ and $c d t R$ was detected in exponential and stationary phases by qPCR. Expression of both genes was detected in all but three of the isolates (Table 1): in strain 765, a NAP1/toxinotype IX isolate, no expression of 
(a)

5' TGAAATACATAGTTATAACTATTTTTATCACGTATAAGAATAAAAATTCCAGAAAATCTTTAT TATCTAAGTTAATATAAAACATATTATTTAATATAAATAAATTTTTTATAATAATATATTGATT

TAATTTACTTTATTATTGTAAAC TATAAGTATAAGATATACTATATAAACAAGCATAAATATAC $-10$

CTTAATTCTAACTATCCTTAATTTTATTTATCTCCTAGTGTTATTTTACGTATTTTTTATAAAA

TACTITTAGTTTTTTTACTTTTGAATTTTTTATAGGAGGGTATTTAGTG 3 '

$$
\text { SD start }
$$

(b)

5 ATCCTCTATATAATAATCGTTTTGTGAAAAAACACATAATGACTATATCATATACAATTTCT

ATtAGACATTGCAACCATTGTTCCTAGATTATGATTCAACAAGATAGCTCATTAAATCATAAAA

GTATGAAATAGTCTCTTTAAAATATTCAGTTGTTATTTTGTACTGACATATCATATAAATACAT

ATTTTTATGATATATAGTTACATATTTTATGAAATTTATATAAAAAAтTCTTATTTAGATTATA

TAATCTAAATAAATTAAAGTCAAGAGTTAATTAAACTAATATTGGGAGGGAGAAAAATG 3'

SD start

(c)

\begin{tabular}{|c|c|}
\hline-35 & \\
\hline ATATTGATTTAATTTACTTTATTATTGTAAACTATAAGTAT & $c d t R$ \\
\hline CAGTTGTTATTTTGTACTGACATATCA.... TATAAATAC & $c d t A$ \\
\hline ATGTTTACAAATTACTATCAGACAAT . . . . СтCСTTATC & tcdA \\
\hline AAGTTTACATATTTATTTCAGACAAC..... GTCTTTATT & $t c d B$ \\
\hline AAGTTTACATAATTATTTAATAATTA..... TGTAATTGT & tcdR1 \\
\hline GTTTTTACA ATACTTTATTAAТАTAА..... AGTTTA TTG & $\operatorname{tcdR2}$ \\
\hline--- TATAAAA $------A---------\ldots$ TAAAAA --- & $\operatorname{tcd} B 1$ \\
\hline-- TTAGCA -------- AAA $--\mathrm{T}--\ldots \ldots$ TATAGT --- & $\operatorname{tcd} B 2$ \\
\hline --GTTCACATCCTCCACCTAAAGCAAATCCG. TTTACAGCA & Clostridial antigen \\
\hline ---TTGTCATATACTGTATAATATTTA. . . TATAATA-- & la \\
\hline$-\overline{\text { TTGACA }}----------------------\overline{\text { TATAAT }}---$ & $\sigma^{70}$ conser \\
\hline
\end{tabular}

Fig. 2. Model of the putative $c d t R$ (a) and $c d t A$ (b) promoter regions in $C$. difficile, and alignment of these promoters with $C$. difficile promoters from the genes encoded within the pathogenicity locus, a clostridial antigen, C. perfringens iota toxin $\mathrm{A}$ promoter and the $\sigma^{70}$ consensus sequence (c). The start codons are labelled as 'start' and are in bold. The putative ShineDalgarno site (SD; ribosome-binding site) is boxed. The putative -10 and -35 promoter elements are in bold, underlined and labelled. The underlined sequence labelled with an asterisk indicates the putative -45 Gram-positive consensus sequence commonly found upstream of the -35 promoter element. Dashes indicate the presence of nucleotides and dots indicate the absence of additional nucleotides. In (c), data are from the following sources: $t c d A, t c d B$, tcdR1 and tcdR2 from Mani et al. (2002); $t c d B 1$ and $t c d B 2$ from Song \& Faust (1998); clostridial antigen from Dailey \& Schloemer (1988); la from Perelle et al. (1993); and $\sigma^{70}$ consensus sequence from Harley \& Reynolds (1987).

either gene was detected by $\mathrm{qPCR}$; in strain 478 , a toxinotype IV isolate, only expression of $c d t R$ was detected; and only $c d t A$ expression was detected in strain 744, a ribotype 078 isolate (data not shown).

\section{Phylogenetic analysis}

Isolates 478, 98 and 744 were the most divergent from the group based on the overall CDTloc sequence and the most 
closely related to each other (Fig. 3a). Isolates 98 and 744 shared the same ribotype, ribotype 078 , and isolate $478 \mathrm{had}$ a unique ribotype (Fig. 3b).

The three ribotype 027 strains (16, 978 and 70) all showed high levels of sequence identity within each gene and promoters, with the most variation detected in the $c d t A$ gene. Within the $c d t A$ gene, there was an SNP and a triple nucleotide polymorphism in strain 16 compared with 978 and 70. Isolates 978 and 70 were identical throughout the CDTloc. The amino acid sequence of strain 16 differed by one amino acid at position 315; this isolate had a valine where the other 027 strains had an asparagine. These isolates are all closely related, as illustrated by the dendrogram (Fig. 3a).

The remaining four isolates belonged to three different ribotypes and two different toxinotypes. Isolates 667 and 765 were closely related despite being from different ribotypes and toxinotypes, but did share the same pulsotype. Isolates 421 and 959 also grouped together and shared both the same ribotype and toxinotype.

\section{Analysis of the cdtR SNP by pyrosequencing}

Pyrosequencing of the $c d t R$ gene in $86 C$. difficile isolates revealed that the truncating $c d t R \mathrm{SNP}$ was restricted to toxinotype $\mathrm{V}$ isolates. It was present in 44 of 57 (77\%) of these isolates. The mutation was present in 17 of 30 (57\%) strains isolated before 2008 and 27 of $27(100 \%)$ strains isolated during or after 2008. Of these isolates, 22 of 57 $(38.5 \%)$ were from human sources and 12 of these $(54.5 \%)$ had the mutation; the remaining 35 (61.5\%) were from non-human sources (including animal and environmental) and 31 of these had the mutation (88.6\%). The remaining 29 strains were categorized into six different ribotypes, including 22 ribotype 027 isolates, but the truncating mutation was not present in any. While there was an association between both year $(P=0.0009)$ and species $(P=0.0129)$ of origin using univariable analysis, presence of the mutation was associated with year $(P=0.0164)$, not animal versus human origin $(P=0.147)$, by nominal logistic regression.

\section{DISCUSSION}

The $C$. difficile binary toxin is a potentially important virulence factor in the pathogenesis of CDI. Here, we sought to improve the understanding of $c d t A$ and $c d t R$ regulation by analysing promoter sequences for specific features, revealing several features common to other bacteria and suggesting that regulation of both genes occurs by a $\sigma^{70}$-like sigma factor. Analysis of full CDT loci sequences revealed a truncating mutation in the $c d t R$ gene of a ribotype 078 isolate. This mutation may result in a

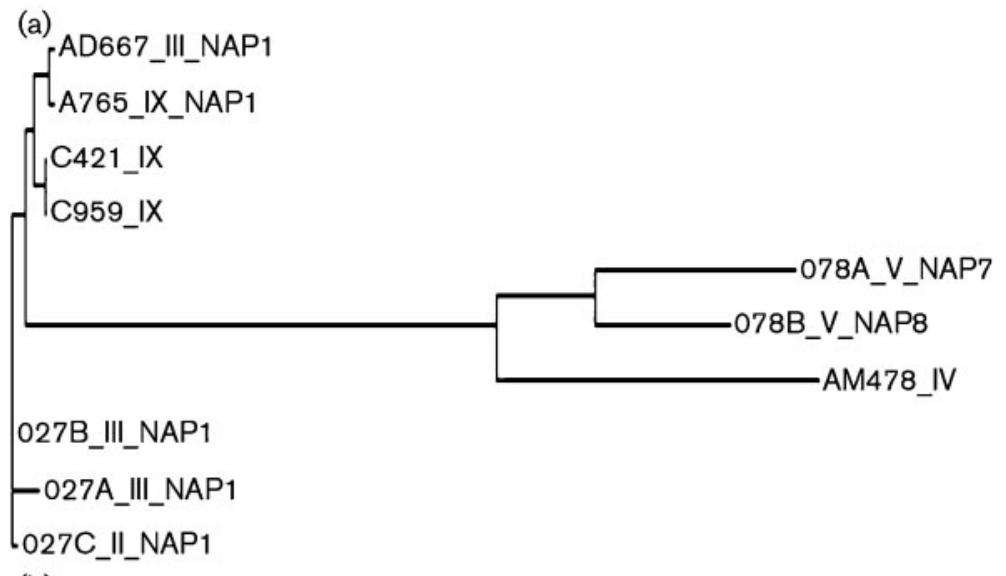

(b)

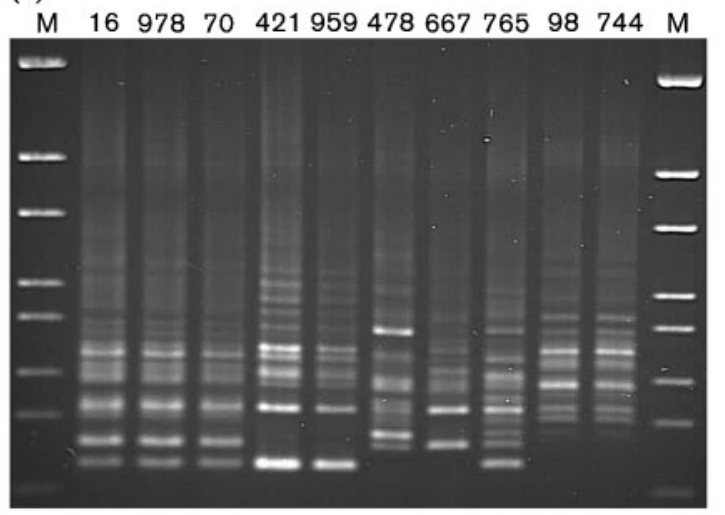

Fig. 3. (a) Phylogram generated from complete binary toxin loci sequences of the ten $C$. difficile isolates (http://www.ebi.ac.uk/Tools/ msa/clustalw2/). Roman numerals indicate toxinotype and the North American pulsotype (NAP) is indicated if known. (b) Ribotype patterns of the ten $C$. difficile isolates. $M$, $100 \mathrm{bp}$ ladder; 16, 027A_III_NAP1; 978, 027B_III_NAP1; 70, 027_III_NAP1; 421, C421_IX; 959, C959_IX; 478, AM478_IV; 667, AD667_IIINAP1; 765, A765_IX_NAP1; 98, 078A_V_NAP7; 744, 078B_V_NAP8. 
non-functional CdtR, yet expression of $c d t A$ in the 078 isolate was still detected suggesting that this regulator is not essential for binary toxin gene expression. The truncating $c d t R$ SNP was also reported by Bouvet \& Popoff (2008) and found to be restricted to toxinotype $\mathrm{V}$ isolates with ribotype profiles similar to ribotype 078 . The mutation was only found in several non-ribotype 078 toxinotype $\mathrm{V}$ isolates, suggesting that the mutation is more broadly associated with toxinotype $\mathrm{V}$, not just ribotype 078 . Bouvet \& Popoff (2008) also demonstrated reduced, but not eliminated, $c d t A$ activity in strains with the $c d t R$ mutation, furthering our assertion that CdtR is not necessary for binary toxin production. This mutation may have emerged recently as it is more often found in more recently obtained strains. Interestingly, this temporal association was present in both human and animal/food animal strains and the apparent emergence of this mutation in both populations gives further support to an association between human, food and food animal strains.

Full CDTloc sequences of ten isolates were analysed and phylogenetic analysis was performed. Ribotype 027 strains were expected to share high sequence identity as strains of this ribotype have been demonstrated to share high overall relatedness (He et al., 2010). Only two polymorphisms were found in the ribotype 027 isolates. This supports the high overall relatedness of this ribotype, although the small sample size must be considered. No significant difference in the number of polymorphisms between $c d t A$ and $c d t B$ was detected, which is in contrast with Pituch et al. (2005) who reported a higher number of polymorphisms in the $c d t B$ gene.

Consistent with other reports showing genetic diversity of ribotype 078 (He et al., 2010; Griffiths et al., 2010), ribotype 078 isolates were the most divergent in their CDT locus. Ribotype 078 was reported to be an outlier based on multilocus sequence typing data and was suggested to have emerged as a genetically distinct clade (Griffiths et al., 2010). Interestingly, the toxinotype IV strain grouped together with the ribotype 078 isolates. The reason for and relevance of this finding are unclear since limited information is available concerning the epidemiology of toxinotype IV strains. The separate grouping of 027 and 078 isolates was not unexpected given the distinct nature of these two ribotypes, but suggests that acquisition of the CDT locus in these two groups was not a recent event.

A third group included four strains from three different ribotypes and two different toxinotypes. Unsurprisingly, the three isolates belonging to toxinotype IX were grouped together; however, a toxinotype III/NAP1 isolate was also included. Therefore, although there appears to be general concordance between ribotype and CDT locus sequence relatedness, this is not absolute.

$c d t A$ and $c d t R$ promoter sequence analysis revealed the presence of several common promoter elements (Fig. 2a, b). Alignments of the $C$. difficile $c d t A$ and $c d t R$ promoters with the $\sigma^{70}$ consensus sequences show shared nucleotides at the -10 and -35 sites (Fig. $2 \mathrm{c}$ ). This suggests that the transcription of genes in the CDTloc may be initiated by a $\sigma^{70}$-like sigma factor. The absence of -35 and -10 consensus sequences upstream of the $c d t B$ start codon was not unexpected since the Clostridium perfringens $\mathrm{Ib}$ promoter also lacks these elements (Perelle et al., 1993). The presence of Shine-Dalgarno sequences, however, suggests that $c d t A$ and $c d t B$ are transcribed monocistronically but translated as two separate proteins. Polymorphisms were identified in each of the binary toxin locus genes but none was predicted to affect secondary structure or function of the protein with the exception of the truncating mutation in $c d t R$.

Analysis of amino acid sequences revealed that the predicted molecular masses for each of the proteins were generally consistent between strains with minor differences reflecting various polymorphisms. Although isoelectric point variations were present, the differences were probably insignificant and would not affect protein solubility or function with the exception of the predicted truncated $\mathrm{CdtR}$ in strain 744 . The variations in amino acid sequences, predicted molecular mass and isoelectric points reflect the heterogeneity of the sequences between isolates. Signal sequences in CdtA and $\mathrm{CdtB}$ were expected as these proteins are predicted to be exported. The absence of a signal sequence in CdtR was also expected as it is thought to regulate gene expression and is predicted to be retained in the cytoplasm.

Response regulators similar to CdtR have been shown to consist of two domains. The N-terminal domain gets phosphorylated by a sensor kinase in response to stimuli. This domain then induces a conformational change in the DNA-binding $C$ terminus. Since the amino acids involved in DNA binding are predicted to occur in the C-terminal domain, they probably would not be translated if CdtR was truncated at 107 amino acids (Nikolskaya \& Galperin, 2002). This suggests that a truncated CdtR might be nonfunctional as a DNA-binding positive regulator of $c d t A B$. The role of the predicated truncated CdtR, however, would need to be assessed in vitro to confirm its functionality. Whether this mutation confers some sort of benefit to the strains that have acquired it remains unknown but we can speculate that it might confer a fitness advantage since there appears to be an increasing number of ribotype 078 strains with this mutation over time and this ribotype is increasing in prevalence amongst CDI cases.

As expected, $c d t A$ and $c d t R$ expression was detected in most isolates assessed, with three exceptions. No obvious sequence variation to explain the lack of expression in strain 765 was detected in the CDTloc and both $c d t A$ and $c d t R$ primers successfully amplified genomic DNA, indicating that variations at the primer sites inhibiting cDNA amplification were not the cause of this result. This suggests that the presence of the binary toxin genes does not necessarily equate to production of the toxin protein. Further supporting this, no $c d t A$ expression was detected in 
478, the toxinotype IV isolate, despite detection of $c d t R$ expression. In contrast, no expression of $c d t R$ but expression of $c d t A$ was detected in the 744 isolate despite the truncating $c d t R$ mutation. The $9 \mathrm{nt}$ insertion in the region upstream of $c d t R$ in the 478 and 744 isolates was not expected to have an effect on expression, but the 744 isolate displayed no $c d t R$ expression whereas expression was still detected in 478 . This suggests that the insertion may lie outside of the active promoter region.

Comparisons of expression levels between strains were not made since only a moderately stable reference gene was used (Metcalf et al., 2010) and a more comprehensive evaluation of expression should be performed using validated, stable reference genes. Reference genes need to be validated in each strain used because the heterogeneity of the species has demonstrated the loss of stability of specific reference genes between strains (Metcalf et al., 2010).

Assessment of the functionality of truncated CdtR and comparison of $c d t A$ expression levels in a wild-type and a strain with the G322T $c d t R$ mutation may provide further insight into the role of the regulator. Although analysis of binary toxin loci did identify various promoter features and reveal a truncating $c d t R$ mutation restricted to toxinotype $\mathrm{V}$ isolates, many questions still remain as to the mechanisms of regulation of the CDT genes and their role in pathogenesis.

\section{ACKNOWLEDGEMENTS}

We thank Drs Michael Mulvey and George Golding for the generous gift of six C. difficile ribotype 078 isolates.

\section{REFERENCES}

Bidet, P., Barbut, F., Lalande, V., Burghoffer, B. \& Petit, J. C. (1999). Development of a new PCR-ribotyping method for Clostridium difficile based on ribosomal RNA gene sequencing. FEMS Microbiol Lett 175, 261-266.

Bouvet, P. J. \& Popoff, M. R. (2008). Genetic relatedness of Clostridium difficile isolates from various origins determined by triple-locus sequence analysis based on toxin regulatory genes $t c d C$, $t c d R$, and $c d t R$. J Clin Microbiol 46, 3703-3713.

Carter, G. P., Lyras, D., Allen, D. L., Mackin, K. E., Howarth, P. M., O'Connor, J. R. \& Rood, J. I. (2007). Binary toxin production in Clostridium difficile is regulated by CdtR, a LytTR family response regulator. J Bacteriol 189, 7290-7301.

Dailey, D. \& Schloemer, R. (1988). Identification and characterization of Clostridium difficile promoter element that is functional in Escherichia coli. Gene 70, 343-350.

Gasteiger, E., Hoogland, C., Gattiker, A., Duvaud, S., Wilkins, M. R., Appel, R. D. \& Bairoch, A. (2005). Protein identification and analysis tools on the ExPASy Server. In The Proteomics Protocols Handbook, pp. 571-607. Edited by J. M. Walker. Totowa, NJ: Humana Press.

Gerber, M., Walch, C., Löffler, B., Tischendorf, K., Reischl, U. \& Ackermann, G. (2008). Effect of sub-MIC concentrations of metronidazole, vancomycin, clindamycin and linezolid on toxin gene transcription and production in Clostridium difficile. J Med Microbiol 57, 776-783.

Govind, R., Vediyappan, G., Rolfe, R. D., Dupuy, B. \& Fralick, J. A. (2009). Bacteriophage-mediated toxin gene regulation in Clostridium difficile. J Virol 83, 12037-12045.

Griffiths, D., Fawley, W., Kachrimanidou, M., Bowden, R., Crook, D. W., Fung, R., Golubchik, T., Harding, R. M., Jeffery, K. J. M. \& other authors (2010). Multilocus sequence typing of Clostridium difficile. J Clin Microbiol 48, 770-778.

Gülke, I., Pfeifer, G., Liese, J., Fritz, M., Hofmann, F., Aktories, K. \& Barth, H. (2001). Characterization of the enzymatic component of the ADP-ribosyltransferase toxin CDTa from Clostridium difficile. Infect Immun 69, 6004-6011.

Harley, C. B. \& Reynolds, R. P. (1987). Analysis of E. coli promoter sequences. Nucleic Acids Res 15, 2343-2361.

He, M., Sebaihia, M., Lawley, T. D., Stabler, R. A., Dawson, L. F., Martin, M. J., Holt, K. E., Seth-Smith, H. M., Quail, M. A. \& other authors (2010). Evolutionary dynamics of Clostridium difficile over short and long time scales. Proc Natl Acad Sci U S A 107, $7527-7532$.

Just, I., Fritz, G., Aktories, K., Giry, M., Popoff, M. R., Boquet, P., Hegenbarth, S. \& von Eichel-Streiber, C. (1994). Clostridium difficile toxin B acts on the GTP-binding protein Rho. J Biol Chem 269, 10706-10712.

Just, I., Selzer, J., von Eichel-Streiber, C. \& Aktories, K. (1995). The low molecular mass GTP-binding protein Rho is affected by toxin A from Clostridium difficile. J Clin Invest 95, 1026-1031.

Karlsson, S., Burman, L. G. \& Åkerlund, T. (2008). Induction of toxins in Clostridium difficile is associated with dramatic changes of its metabolism. Microbiology 154, 3430-3436.

Mani, N., Lyras, D., Barroso, L., Howarth, P., Wilkins, T., Rood, J. I., Sonenshein, A. L. \& Dupuy, B. (2002). Environmental response and autoregulation of Clostridium difficile TxeR, a sigma factor for toxin gene expression. J Bacteriol 184, 5971-5978.

Martin, H., Willey, B., Low, D. E., Staempfli, H. R., McGeer, A., Boerlin, P., Mulvey, M. \& Weese, J. S. (2008). Characterization of Clostridium difficile strains isolated from patients in Ontario, Canada, from 2004 to 2006. J Clin Microbiol 46, 2999-3004.

McDonald, L., Killgore, G., Thompson, A., Owens, R., Jr, Kazakova, S., Sambol, S., Johnson, S. \& Gerding, D. (2005). An epidemic, toxin gene-variant strain of Clostridium difficile. N Engl J Med 353, 24332441.

Metcalf, D., Sharif, S. \& Weese, J. S. (2010). Evaluation of candidate reference genes in Clostridium difficile for gene expression normalization. Anaerobe 16, 439-443.

Nikolskaya, A. N. \& Galperin, M. Y. (2002). A novel type of conserved DNA-binding domain in the transcriptional regulators of the AlgR/ AgrA/LytR family. Nucleic Acids Res 30, 2453-2459.

Perelle, S., Gibert, M., Boquet, P. \& Popoff, M. R. (1993). Characterization of Clostridium perfringens iota-toxin genes and expression in Escherichia coli. Infect Immun 61, 5147-5156.

Perelle, S., Gibert, M., Bourlioux, P., Corthier, G. \& Popoff, M. R. (1997). Production of a complete binary toxin (actin-specific ADP-ribosyltransferase) by Clostridium difficile CD196. Infect Immun 65, 1402-1407.

Pituch, H., Kreft, D., Obuch-Woszczatynski, P., Wultańska, D., Meisel-Mikołajczyk, F., kuczak, M. \& van Belkum, A. (2005). Clonal spread of a Clostridium difficile strain with a complete set of toxin A, toxin B, and binary toxin genes among Polish patients with Clostridium difficile-associated diarrhea. J Clin Microbiol 43, 472-475.

Schwan, C., Stecher, B., Tzivelekidis, T., van Ham, M., Rohde, M., Hardt, W. D., Wehland, J. \& Aktories, K. (2009). Clostridium 
difficile toxin CDT induces formation of microtubule-based protrusions and increases adherence of bacteria. PLoS Pathog 5, e1000626.

Song, K. P. \& Faust, C. (1998). Molecular analysis of the promoter region of the Clostridium difficile toxin $\mathrm{B}$ gene that is functional in Escherichia coli. J Med Microbiol 47, 309-316.
Stare, B. G., Delmée, M. \& Rupnik, M. (2007). Variant forms of the binary toxin CDT locus and tcdC gene in Clostridium difficile strains. J Med Microbiol 56, 329-335.

Stubbs, S., Rupnik, M., Gibert, M., Brazier, J., Duerden, B. \& Popoff, M. (2000). Production of actin-specific ADP-ribosyltransferase (binary toxin) by strains of Clostridium difficile. FEMS Microbiol Lett 186, 307-312. 